\title{
Extraction of Sleep-Spindles from the Electroencephalogram (EEG)
}

\author{
Allan Kardec Barros \\ Bio-mimetic Control Research Center, RIKEN, \\ 2271-130 Anagahora, Shimoshidami, Moriyama-ku, Nagoya 463, Japan \\ Roman Rosipal, Mark Girolami \\ Department of Computing and Information Systems, University of Paisley \\ Paisley, PA1 2BE, Scotland \\ Georg Dorffner \\ Dept. of Medical Cybernetics and Artificial Intelligence, University of Vienna \\ Freyung 6/2, A-1010 Vienna, Austria \\ and \\ Austrian Research Institute for Artificial Intelligence \\ Schottengasse 3, A-1010 Vienna, Austria \\ Noboru Ohnishi \\ Bio-mimetic Control Research Center, RIKEN, \\ 2271-130 Anagahora, Shimoshidami, Moriyama-ku, Nagoya 463, Japan
}

\begin{abstract}
Independent component analysis (ICA) is a powerful tool for separating signals from their observed mixtures. This area of research has produced many varied algorithms and approaches to the solution of this problem. The majority of these methods adopt a truly blind approach and disregard available $a$ priori information in order to extract the original sources or a specific desired signal. In this contribution we propose a fixed point algorithm which utilizes a priori information in finding a specified signal of interest from the sensor measurements. This technique is applied to the extraction and channel isolation of sleep spindles from a multi-channel electroencephalograph (EEG).
\end{abstract}

\section{Introduction}

Sleep spindles are particular EEG patterns which occur during the sleep cycle at 11.5 to $15 \mathrm{~Hz}$, and are used as one of the features to classify the varying stages of sleep. The visual detection of sleep spindles is particularly difficult when multi-channel EEG recordings are used due to inter-channel degradation of the sleep-spindle signatures and other sources of unwanted signals which can be regarded simply as noise.

To aid the manual and possibly automatic detection of sleep spindle activity, it would be desirous to isolate the spindles in a single channel of EEG measurements. This could be possible by the application of a suitable form of the independent component analysis (ICA) transform for the extraction of an information bearing signal. 
Preliminary work on sleep spindle isolation has been reported in Rosipal et al. [8]. ICA appeared recently as a promising technique for separating independent sources in biomedical signal processing $[1,6,9]$. ICA is based on the following principle. Assuming that the original (or source) signals have been linearly mixed in some otherwise unknown manner, and that these mixed signals are available as observations, ICA is a single neuron network which seeks to find in a blind manner a linear combination of the mixed signals which recovers the original source signals, possibly re-scaled and randomly arranged in the outputs.

However, extracting all the independent signals from, for example, a set of EEG measurements can be a lengthy and computationally demanding process. Therefore, in some cases, it may be important to extract only the subset of desired components however these may be defined. In the case under consideration, that of isolation of sleep spindles, we would seek to extract only the sleep spindles for further possible manipulation. To achieve this desired signal extraction we propose the use of a single neuron deflation algorithm along with the utilization of the $a$ priori information available about the signal of interest. In fact, the deflation approach is important because we can extract only one independent component out of the measured signals, instead of separating all of them at once. However, as mentioned previously, the independent components are extracted in a non-deterministic manner, thus any one of the signals could potentially appear as the first extracted source. In solving this problem, we use the a priori information available about the desired signal as the input to a standard Wiener filter to initialize the signal extraction algorithm.

In the literature, Rosipal et al. [8] were the first to suggest that the application of general ICA algorithms could minimize the channel overlap in the transformed EEG, thus possibly isolating the sleep-spindle patterns. Our objective here is to enhance this isolation and possible subsequent classification by applying an ICA technique which will enforce the extraction of the sleep spindles alone. This then ensures that the electroencephalographer (or some automated classifier) would have only the sleep spindles at hand, after processing the measured EEG with the proposed new ICA algorithm.

\section{Second Order Statistics}

Let us first define the problem in a framework that will be used throughout this paper. Consider $n$ statistically mutually independent signals $\mathbf{s}(k)=\left[s_{1}(k), s_{2}(k), \ldots, s_{n}(k)\right]^{T}$ arriving at $n$ electrodes (or another type of receiver). Each electrode receives an unknown linear combination of the signals. For simplicity, we will drop the iteration index $k$ and only use when necessary, then we have $\mathbf{s}=\left[s_{1}, s_{2}, \ldots, s_{n}\right]^{T}$. The mixed signals $\mathbf{v}$ are thus given by $\mathbf{v}=\mathbf{A s}$, where $\mathbf{A}$ is an $n \times n$ invertible matrix ${ }^{1}$. We assume that we can observe only the mixture v. Moreover, without loss of generality, let $\mathbf{x}=\mathbf{M v}$, so that $E\left[\mathbf{x x}^{T}\right]=\mathbf{I}$. In practice, this operation will speed up the convergence of the algorithm to be presented later, in addition to producing a simpler form of the updating technique.

Our purpose is to find from the mixed observation vector $\mathbf{x}$ one given component

\footnotetext{
${ }^{1}$ This is a simplified model of the more general linear mixing model where there may be modulation of the source and observation dimensions and sensor noise also may be included.
} 
$s_{i}$ of the source signal $\mathbf{s}$, using some $a$ priori information included in a signal $d$, correlated with $s_{i}$, i.e., $E\left[d s_{i}\right] \neq 0$. In carrying this out, we use all the components of the input vector $\mathbf{x}$ so that we have $u=\mathbf{w}^{T} \mathbf{x}$, where $\mathbf{w}$ is a weight vector to be estimated by the adaptive algorithm, $d$ is the reference signal and the error is given by $\varepsilon=d-u$. The weights are updated by the minimization of the mean squared error (MSE) given by $E\left[\varepsilon^{2}\right]$.

One can find easily that the optimum weight, which minimizes the MSE is $\mathbf{w}_{*}=$ $\mathbf{R}^{-1} \mathbf{P}$, where $\mathbf{R}$ and $\mathbf{P}$ are defined as the correlation matrix of $\mathbf{x}$ and the cross correlation matrix of $\mathbf{x}$ and $d$ respectively. Due to the spatial whitening of the observation signals $E\left[\mathbf{x x}^{T}\right]=\mathbf{I}$ the Wiener solution optimum weight vector is then given by

$$
\mathbf{w}_{*}=\mathbf{R}^{-1} \mathbf{P}=E[d \mathbf{x}] .
$$

Barros et al. [1] have shown that in the presence of a reference signal, second order statistics are enough to provide separation of the source signals.

\section{ICA for Biomedical Signal Processing}

The final objective of ICA is also to separate signals, under the assumption that they are mutually independent, in other words, the joint probability density of the source signals is the product of the marginal densities of the individual sources, $p(\mathbf{s})=$ $\prod_{i=1}^{M} p\left(s_{i}\right)$. The difference with the method in the previous section is that most of the algorithms in the literature were proposed for separating the signals in a blind manner. Within this framework, ICA algorithms find a linear combination of the elements of $\mathbf{x}$ which gives the most independent components as the output. Usually this output is found using a matrix $\hat{\mathbf{W}}$, so that the elements of $\mathbf{z}=\hat{\mathbf{W}} \mathbf{x}$ are approximately mutually independent.

To find the matrix $\hat{\mathbf{W}}$, many different algorithms have been proposed in the literature. However, we are interested in the ability to extract or remove some given signal from a number of measured signals. Therefore, instead of finding an $n \times n$ matrix $\hat{\mathbf{W}}$ and from the output $\mathbf{z}=\hat{\mathbf{W}} \mathbf{x}$ pick up the desired signal (as in [7]), we are interested in finding only one component $z_{i}$ of $\mathbf{z}$. Therefore, this component is given by $z_{i}=\hat{\mathbf{w}}_{i}^{T} \mathbf{x}$, where $\hat{\mathbf{w}}_{i}$ is one of the rows of $\hat{\mathbf{W}}$.

Dropping the index $i$ for the purposes of exposition clarity, in the estimation of $\hat{\mathbf{w}}$, we combine the following updating rules:

$$
\begin{aligned}
& \hat{\mathbf{w}}_{k+1}=E\left[\mathbf{x}\left(\hat{\mathbf{w}}_{k}^{T} \mathbf{x}\right)^{3}\right]-3 \hat{\mathbf{w}}_{k}, \\
& \hat{\mathbf{w}}_{k+1}=\mathbf{R}_{\mathbf{x} \tilde{\mathbf{z}}} \mathbf{R}_{\tilde{\mathbf{z}} z},
\end{aligned}
$$

where $\mathbf{R}_{\mathbf{x} \tilde{\mathbf{z}}}=E\left[\mathbf{x} \tilde{\mathbf{z}}^{T}\right]$ and, $\tilde{\mathbf{z}}(k)=[z(k-1) \cdots z(k-L)]^{T}$, and $L$ is a delay. In the above equations, (2) is based on finding the extrema of the transformed output kurtosis [5]. As it may fail or show slow convergence in the case of low normalized values of kurtosis, we use (3) to update the algorithm [3]. It has been found that the combination of the two adaptive steps is a very efficient solution for the problem of blind source separation. 


\subsection{Proposed Algorithm}

From the reasoning in the previous section, we propose the following fixed-point algorithm:

- Perform a principal component analysis (PCA) decomposition on the sample covariance matrix of the observation vectors $\mathbf{v}$ and project $\mathbf{v}$ onto the matrix of normalized eigenvectors $\mathbf{M}$ such that, $\mathbf{x}=\mathbf{M v}$ and $E\left[\mathbf{x x}^{T}\right]=\mathbf{I}$.

- Take the initial vector $\hat{\mathbf{w}}_{0}=E[d \mathbf{x}] /\|E[d \mathbf{x}]\|$. Iteration number $k=1$.

- Update $\hat{\mathbf{w}}$ by, $\hat{\mathbf{w}}_{k+1}=E\left[\mathbf{x}\left(\hat{\mathbf{w}}_{k}^{T} \mathbf{x}\right)^{3}\right]-3 \hat{\mathbf{w}}_{k}$. If the normalized value of the sample kurtosis of $z$ is tending to zero, shift the updating to the kurtosis invariant algorithm

$$
\hat{\mathbf{w}}_{k+1}=\mathbf{R}_{\mathbf{x} \tilde{\mathbf{z}}} \mathbf{R}_{\tilde{\mathbf{z}} z}
$$

- Divide $\hat{\mathbf{w}}_{k}$ by its norm and update $k=k+1$.

- Test if $\left\|\hat{\mathbf{w}}_{k}-\hat{\mathbf{w}}_{0}\right\|<\zeta$, otherwise, change the current weight to the Wiener one, added to a small random deviation. This step is important to guarantee that the solution is spatially close to the Wiener one.

- Repeat the last three above steps until $\left\|\hat{\mathbf{w}}_{k+1}^{T} \hat{\mathbf{w}}_{k}\right\|$ approaches 1 (up to a small error $)^{2}$.

\subsection{Signal Enhancement/Elimination}

After obtaining the output $z=\mathbf{w} \hat{\mathbf{M v}}$ using the algorithm described above, one may be interested either in keeping this signal, or in removing it from the sensors for subsequent manipulation or analysis. In order to accomplish this last option, we can simply use the Wiener filter as proposed above. This can be carried out by estimating the signal $z$ using the previous ICA method, and computing its contribution to each element of $\mathbf{x}$. Thus, one can either have a vector of the contribution of $z$ to each sensor as $\mathbf{y}=\mathbf{b} z$, or the sensors with $z$ eliminated from it given by $\tilde{\mathbf{y}}=\mathbf{v}-\mathbf{b} z$. From (1), we find that the elements of $\mathbf{b}$ are estimated by

$$
\mathbf{b}=E[\mathbf{v} z] .
$$

\section{Results}

A seven minutes recording of 18 channels of EEG (Fp1, F8, F4, Fz, F3, F7, T4, C4, $\mathrm{Cz}, \mathrm{C} 3, \mathrm{~T} 3, \mathrm{~T} 6, \mathrm{P} 4, \mathrm{Pz}, \mathrm{P} 3, \mathrm{~T} 5, \mathrm{O} 2, \mathrm{O} 1)$ was used as the input data for the following experiments to demonstrate the utility of the proposed approach to biomedical signal processing. Electrodes were placed according to the international 10-20 system. The data were digitized with a sampling rate of $102.4 \mathrm{~Hz}$.

\footnotetext{
${ }^{2}$ The MATLAB code for this algorithm is available upon request. Or, if the reader is interested in a version without a reference input, refer to the site in [2].
} 
The measured signals were then filtered by a butterworth band-pass filter between 10 and $20 \mathrm{~Hz}$. The signals were passed forward and backward through the filter to avoid phase distortion.

Channels HEO and $\mathrm{Cz}$ of the EEG were chosen as the reference inputs to extract the sleep spindles. The results are shown in Fig. 1 for a window of 10 seconds it is clear that the sleep spindles have been extracted and therefore isolated within this single output channel. Notice that the outputs are already scaled in relation to the references using (5).
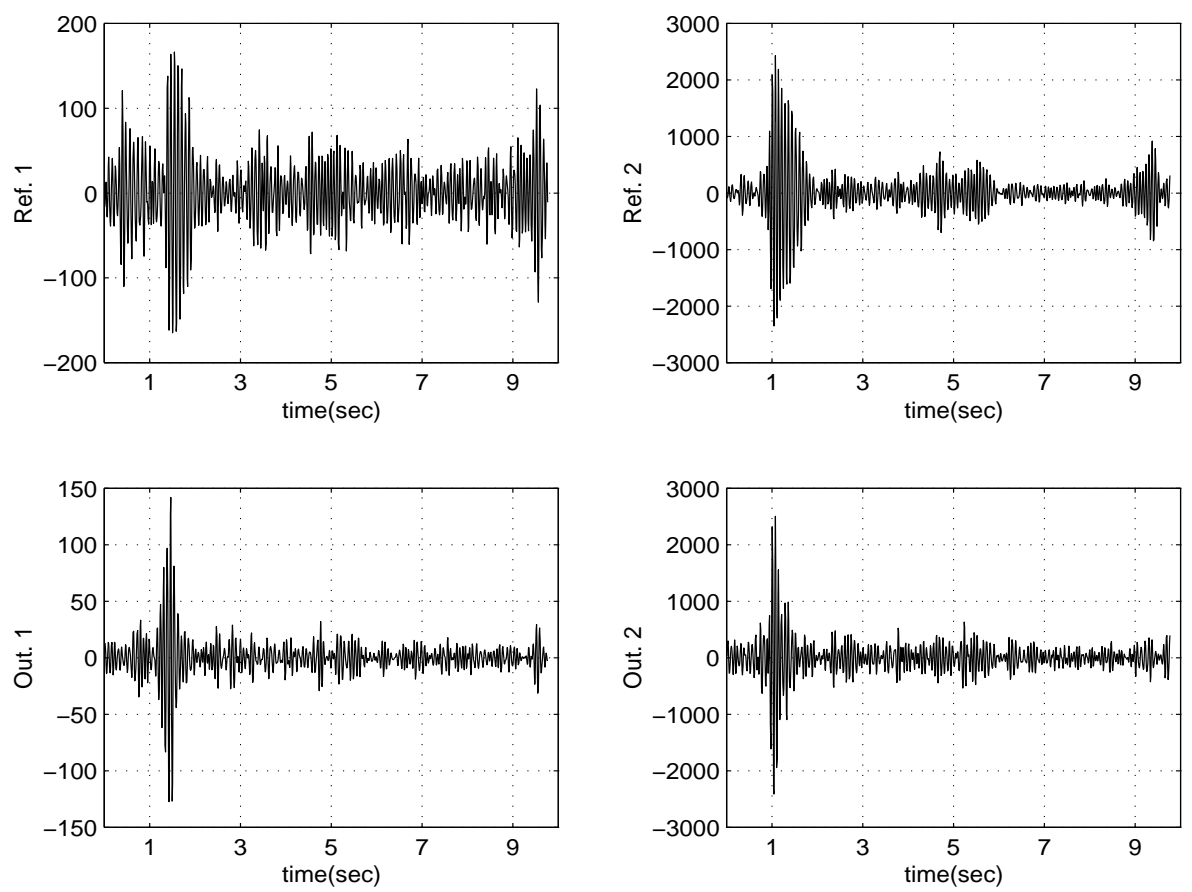

Figure 1: Example of two sleep spindles extracted from the raw data.

\section{Conclusions}

In this paper we have introduced an algorithm which will prove useful in biomedical signal processing where a specific underlying signal requires to be extracted from the possibly noisy multi-channel recordings. This algorithm is a modified version of the that originally developed in [1]. It is clear that this method is suitable for the extraction of independent components from the measured EEG. Experimental evidence of the algorithm's ability to extract pre-specified signals has been given using multi-channel EEG. The algorithm worked efficiently in extracting sleep spindles which were distributed throughout the measurement channels. 


\section{Acknowledgments}

The authors thanks Prof. Zeitlhofer for providing EEG data set which was recorded at the Clinic of Neurology at the University of Vienna. This work was done as part of the Biomed-2 project BMH4-CT97-2040 SIESTA, sponsored by the EU Commission. $\mathrm{RR}$ is funded by a research grant for the project "Objective Measures of Depth of Anaesthesia"; University of Paisley and Glasgow Western Infirmary NHS trust, and is partially supported by Slovak Grant Agency for Science (grants No. 2/5088/98 and No. 98/5305/468). The Austrian Research Institute for Artificial Intelligence is supported by the Austrian Federal Ministry of Science and Transport.

\section{References}

[1] Barros AK, Vigário R, Jousmaki V, Ohnishi N. Extraction of Event-Related Signals from Multi-channel Bioelectrical Measurements submitted to a journal.

[2] Barros AK, Cichocki A. RICA - Reliable and robust program for Independent Component Analysis. Technical Report and MATLAB program of RIKEN. Web page: http://www.bmc.riken.jp/sensor/allan/RICA or http://go.to/RICA.

[3] Cichocki A, Barros AK. Robust Batch Algorithm for Sequential Blind Extraction of Noisy Biomedical Signals. In: Proceedings of ISSPA 1999, pp 363-366.

[4] Delfosse N, Loubaton P. Adaptive blind separation of independent sources: a deflation approach. Signal Processing 1995; 45:59-83.

[5] Hyvärinen A, Oja E. A fast fixed-point algorithm for independent component analysis. Neural Computation 1997; 9:1483-1492.

[6] Jung T-P, Makeig S, Westerfield M, Townsend J, Courchesne E, Sejnowski T. Independent component analysis of single-trial event related potentials. In: Proceedings ICA 1999, pp 173-179.

[7] Makeig S, Jung T-P, Ghahremani T, Bell AJ, Sejnowski T. Blind separation of event-related brain responses into independent components. In: Proceedings Natl Acad Sci USA 1997, 94:10979-10984.

[8] Rosipal R, Dorffner G, Trenker E. Can ICA improve sleep-spindles detection? Neural Networks World 1998; 5:539-547.

[9] Vigário R, Jousmaki V, Hamalainen M, Hari R, Oja E. Independent component analysis for identification of artifacts in magnetoencephalographic recordings. In: Advances in Neural Information Processing Systems. MIT press, 1997. 